Recepción: 20 / 04 / 2017

Aceptación: 20 / 05 / 2017

Publicación: 15 / 09 / 2017

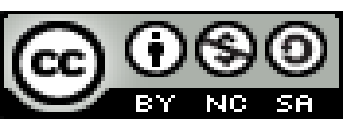

Ciencias de la Educación

Revisión de Literatura

\title{
Desarrollo intelectual en la primera infancia
}

\section{Intellectual development in early childhood \\ Desenvolvimento Intelectual na Primeira Infância}

\author{
Yadira L. Sánchez-Padilla ${ }^{\mathrm{I}}$ \\ ysanchez@utmachala.edu.ec \\ Gerardo X. Peña-Loaiza II \\ gpena@utmachala.edu.ec \\ Rosa M. Salamea-Nieto III \\ rsalamea@utmachala.edu.ec \\ Carlos P. Carpio-Mosquera IV \\ ccarpio@utmachala.edu.ec
}

Correspondencia: ysanchez@utmachala.edu.ec

I. Magister en Psicoterapia Integrativa; Psicóloga Clínica; Universidad Técnica de Machala, Machala, Ecuador.

II. Magister en Terapia Familiar con Mención en Psicoterapia Sistémica; Diploma Superior en Docencia Universitaria; Psicólogo Clínico; Universidad Técnica de Machala, Machala, Ecuador.

III. Magister en Educación Superior; Licenciada en Ciencias de la Educación Mención Psicología Educativa; Psicóloga Clínica; Universidad Técnica de Machala., Machala, Ecuador.

IV . Diplomado Superior en Gerencia de Recursos Humanos; Magister en Psicoanálisis con Mención en Educación; Psicólogo Clínico; Licenciado en Psicología Clínica; Universidad Técnica de Machala, Machala, Ecuador. 


\section{Resumen}

En el presente artículo se hace una breve revisión teórica del Desarrollo Intelectual en la Primera Infancia que tuvo con objetivo caracterizar el desarrollo intelectual en la Primera Infancia y posteriormente señalar los resultados de las investigaciones que se han efectuado en esta área. Se describe el aporte de la teoría piagetiana y de apego en el estudio del desarrollo cognitivo infantil, así como algunas contribuciones representativas de pioneros y actuales investigadores que han influido en el área. En el desarrollo infantil, los tres primeros años de vida son cruciales, en este periodo ocurre la maduración del sistema nervioso central que a su vez está sujeto a los estímulos del medio exterior. La interacción de estos repercute en el área social, la coordinación, el lenguaje, la motricidad y la cognición del infante. Los estudios, realizados en el área de la psicología perinatal, han puesto en evidencia que algunos factores sociodemográficos, afectivos, biológicos, entre otros, pueden mejorar o a su vez perjudicar significativamente el desarrollo cognitivo del infante.

Palabras claves: Piaget; desarrollo infantil; psicología perinatal; primera infancia. 


\begin{abstract}
This article presents a brief theoretical review of Intellectual Development in Early Childhood that aimed to characterize the intellectual development in Early Childhood and then to indicate the results of the research that has been carried out in this area. We describe the contribution of Piagetian and attachment theory in the study of children's cognitive development, as well as some representative contributions of pioneers and current researchers who have influenced the area. In child development, the first three years of life are crucial, during this period the maturation of the central nervous system occurs which in turn is subject to the stimuli of the external environment. The interaction of these affects the social area, coordination, language, motor and cognition of the infant. Studies carried out in the area of perinatal psychology have shown that some sociodemographic, affective, biological factors, among others, may improve or in turn significantly impair the cognitive development of the infant.
\end{abstract}

Key words: Piaget; child development; perinatal psychology; early childhood. 


\section{Introducción.}

En el desarrollo infantil, los tres primeros años de vida son cruciales, en este periodo ocurre la maduración del sistema nervioso central que a su vez está sujeto a los estímulos del medio exterior. La interacción de estos repercute en el área social, la coordinación, el lenguaje, la motricidad y la cognición del infante. En cuanto al desarrollo cognitivo, Piaget considera que se produce por el rol activo del ser cognoscente quien integra de forma dialéctica la subjetividad, sensibilidad y razonamiento lógico en el desarrollo de la inteligencia, que en primer momento es práctica y posteriormente reflexiva. Innumerables investigaciones, especialmente en el área de la psicología perinatal, han puesto en evidencia que algunos factores sociodemográficos, afectivos, biológicos, entre otros, pueden mejorar o a su vez perjudicar significativamente el desarrollo cognitivo del infante. Se resalta de esta manera la importancia de realizar investigaciones que permitan identificar los factores que influyen en el desarrollo cognitivo de la primera infancia y las propuesta correctivas que se pueden considerar.

Bajo este contexto se realiza el presente análisis que tiene como objetivo en primer lugar caracterizar el desarrollo intelectual en la Primera Infancia y posteriormente señalar los resultados de las investigaciones que se han efectuado en esta área. Se selecciona el área intelectual como tema de análisis, por ser tal vez el marco de referencia con más evidencia empírica y por el interés del autor en profundizar en el tema.

\section{Metodología.}

La metodología de investigación empleada para este trabajo fue de tipo documental, en donde fueron utilizadas diferentes referencias bibliográficas y documentales, con el objetivo de seguir profundizando y ampliando los temas sobre el desarrollo intelectual en edad temprana. 


\section{Resultados}

\section{Desarrollo Intelectual en la Infancia}

La extensa obra de Piaget requiere selecciones y recortes a la hora de su abordaje. Su ojo minucioso identificó los modos en que se iba complejizando el niño y se preguntó de qué manera eso podía sintetizarse en diferentes períodos. A partir de ello, es en el año 1956 que Piaget presenta su teoría y en ella concibe el desarrollo cognitivo de los seres humanos como un proceso que se organiza y reorganiza permanentemente y diferencia estas etapas en periodos, sub-períodos y estadios en los cuales de acuerdo a la edad que tenga el niño debe alcanzar ciertos logros hasta adquirir la maduración necesaria que dará paso a la siguiente etapa (Piaget, 1990; Phillips, 1977, Albornoz y Guzmán, 2016)

Es frecuente encontrar en ciertos trabajos una emulación de la teoría piagetiana como de “escalones” que se relacionan de manera no problemática por lo que es importante dejar claro que las etapas que identificó Piaget deben pensarse dialécticamente, es decir, no como "un paso que sucede a otro que lo precede" sino como estructuraciones y desestructuraciones complejas (Duek, 2010).

Piaget (1990) distingue dos periodos en el desarrollo de la inteligencia, uno de ellos es la inteligencia sensoriomotriz, entendida como la capacidad de solucionar problemas sin la necesidad de emplear evocaciones simbólicas (Oiberman, 2006; Stassen, 2009).

Para este autor, la inteligencia sensoriomotriz es un periodo en que "el lactante no presenta todavía ni pensamiento ni afectividad ligada a representaciones que permitan evocar personas $u$ objetos ausentes pero es de importancia especial, porque el niño elabora a ese nivel el conjunto de 
las subestructuras cognoscitivas que servirán de punto de partida a sus construcciones perceptivas e intelectuales ulteriores" (Piaget y Inhelder, 2000, p. 15)

A la teoría del periodo de la inteligencia sensoriomotriz, Piaget la subdividió en seis estadios: adaptaciones sensorio-motrices elementales (Estadío I), los primeros hábitos (Estadío II), las adaptaciones sensorio-motrices intencionales (Estadío III), distinción entre medios y fines (Estadío IV), descubrimiento de los medios nuevos por experimentación activa (Estadío V) e invención de medios nuevos por combinación mental (Estadío VI) (Oiberman et al., 2015).

Como se había anunciado en los apartados anteriores y en base a la teoría piagetiana se describe a continuación varios estudios que se han realizado y han estado orientados a indagar los factores que contribuyen o retardan el desarrollo cognitivo del niño, más aun cuando existe una amplia coincidencia a nivel mundial en cuanto a que los cuidados físicos y una alimentación apropiada, a pesar que son factores necesarios, resultan insuficientes para que los niños alcancen un desarrollo óptimo. En este sentido, Oiberman et al., (2015), realizaron una investigación con el fin de evidenciar el impacto de los desencuentros tempranos en el desarrollo cognitivo en niños alojados en dispositivos convivenciales institucionales, a través de la Escala Argentina de Inteligencia Sensorio Motriz (EAIS). Dicho instrumento examina y valora las estrategias que utiliza el bebé en la resolución de 4 situaciones que se le presentan: exploración de los objetos, búsqueda del objeto desaparecido, utilización de intermediarios y combinación de objetos (Oiberman et al., 2006). En el mencionado estudio se evaluaron las características de los estadios III al VI, presentes en los niños.

Como se esperaba, los resultados evidenciaron una relación dinámica entre el desarrollo cognitivo del niño y los vínculos afectivos, pues entre otros aspectos, de manera especial se notó que 
los niños que presentaron un desarrollo normal se caracterizaban por tener o haber tenido una relación estable con algún familiar o cuidador del centro, lo que redujo probablemente los efectos negativos de ser institucionalizado.

Ortiz, Duelo y Escribano (2013) reconocen la importancia de la presencia de un cuidador en el desarrollo óptimo del infante, según ellos a través de la función de filtro y elaboración que la madre proporciona al niño, este va consiguiendo gradualmente una comprensión de sus propios estados internos, a la vez que comienza a poder regularlos. Además agregan que las experiencias sensoriales del niño se convertirán en percepciones y después en representaciones. Manifiestan también que si el cuidador no cumple esta función reguladora de modo adecuado, el niño experimentará diversas perturbaciones, como la vivencia ulterior de que sus sentimientos son confusos y difíciles de regular. Y finalmente añaden que en los casos más graves, aparecerán más tarde dificultades para diferenciar la fantasía de la realidad, y la realidad psíquica de la física.

Se resalta de esta manera la importancia de los vínculos afectivos del cuidador y el apoyo social en el desarrollo del niño. Como se mencionó anteriormente, el desarrollo del niño implica el aspecto físico, cognitivo y psicosocial. Según Ortiz, Duelo y Escribano (2013) para entender el desarrollo del niño, hay que tener en cuenta la interdependencia entre el desarrollo afectivo, cognitivo y motor, y considerar al ser humano de forma integral, como una unidad biopsicosocial.

El vínculo de dependencia y la relación dinámica entre cada una estas áreas, las convierten en un aspecto importante de atender, así lo ratifica Papalia et al., (2012) al mencionar que "las capacidades físicas y cognoscitivas repercuten en el desarrollo psicosocial, ya que contribuyen en buena medida a la autoestima e influyen en la aceptación social y la elección vocacional” p. 6. 
Es difícil encontrar comportamientos que denoten únicamente afectividad, sin elementos cognitivos y viceversa, pues el niño al percibirse en un ambiente agradable y motivador va a generar nuevos aprendizajes con entusiasmo y ello contribuye a su buen desarrollo, no siendo así, si el niño se encuentra en un ambiente que le generara un bloqueo afectivo (Vargas y Arán, 2014). Por lo tanto, lo emocional o afectivo va a influir en el desarrollo intelectual que tiene el niño, no modificando sus estructuras, pero si estimulando o retrasando su desarrollo cognitivo.

El estudio descrito en el apartado anterior que enfatizó en la importancia del factor afectivo para el desarrollo cognitivo del niño, se complementa con los resultados alcanzados en otro proyecto de investigación que tuvo como objetivo relacionar variables sociodemográficas al riesgo o retraso en el desarrollo cognitivo (Paolini, Santos y Oiberman, 2015), teniendo como antecedente resultados de estudios anteriores que resaltan el valor especial de los agentes sociales y ambientales por sobre los biológicos y hereditarios (Evans y English, 2002; Schonhaut, Maggiolo, Herrera, Acevedo y García, 2008).

El estudio arrojó resultados interesantes como el hecho de constatar que las características del desarrollo infantil propias del segundo año de vida, hacen necesaria la adquisición y consolidación de habilidades para el desarrollo cognitivo del niño (Paolini et al., 2015).

También se pudo revelar que los niños de edad gestacional menor a 40 y especialmente 37 semanas, presentan dificultad en el desarrollo cognitivo. Este resultado coincide con pocos datos encontrados en la literatura científica que han identificado igualmente variaciones cognitivas relacionadas con la edad de gestación.

Finalmente y resaltando la importancia de la investigación desarrollada por Paolini, Santos y Oiberman (2015), se han realizado un número limitado de estudios que analicen el proceso de 
interacción de las conductas parentales y sus efectos en el desarrollo de las competencias cognitivas de los hijos; sin embargo, otro dato interesante que se registró en los resultados del estudio descrito, es la asociación entre el desempleo del padre y las dificultades en el desarrollo cognitivo infantil, corroborando así resultados obtenidos en estudios preliminares que resaltan la importancia de la participación paterna en el desarrollo temprano del niño.

De esta manera, los resultados obtenidos en la investigación realizada por Oiberman y otros (2002, 2005), fueron significativos, en general sus aportes teóricos han estado orientados a diferenciar con exactitud si los niños inician, transitan o finalizan un determinado subestadio y que factores retrasan o promueven este proceso. La EAIS como se pudo constatar en el resultado de la investigación, permite precisar el nivel de las habilidades cognitivas alcanzadas en edades tempranas; al tiempo que se convierte en un instrumento

Del mismo modo, entre otros trabajos que se han realizado para valorar el desarrollo cognitivo del niño, está la Escala Argentina de Dibujo Infantil (EADI), elaborada con el objetivo de proporcionar a los profesionales un instrumento que permita evaluar el desarrollo cognoscitivo y madurativo en niños de dos a seis años a partir del dibujo de la figura humana.

Varias investigaciones han puesto en evidencia el valor del apego en el desarrollo emocional, social y cognitivo del ser humano (Chamorro, 2012; Vargas y Arán, 2014; Oiberman et al., 2015). De acuerdo a la teoría del apego creada por Bowlby (1982), este se refiere a la estrecha y especial relación que puede haber entre el cuidador y el niño (Garrido, 2006; Grimalt y Heresi, 2012). Es así que de la calidad de las relaciones afectivas que establezca el ser humano en los primeros años de vida, dependerá la habilidad de responder a las diversas situaciones que enfrente a lo largo de su existencia (Rodríguez y Oiberman, 2016). 
De varios instrumentos que se han elaborado para evaluar el apego en niños pequeños y su cuidador en edades tempranas, resalta el Procedimiento de la Situación Extraña (Ainsworth, Blehar, Waters y Wall, 1978). Esta escala observacional fue validada en Argentina para ser aplicada en niños pequeños entre 12 y 36 meses de edad con la finalidad identificar el tipo de apego que adopta el niño ante situaciones de estrés (Rodríguez y Oiberman, 2016). Cuando las respuestas sean poco adaptativas, se consideraría tomar medidas preventivas.

El Método de la Situación Extraña además fue empleado por Rodríguez y Oiberman (2011), en un trabajo de investigación que trazó como objetivo observar las conductas de niños de entre 1 y 3 años de edad que son direccionados a mantenerse solos. Con este procedimiento se busca establecer hasta que edad el niño acepta quedarse solo, sin la compañía de su cuidador, siendo esta conducta un signo importante de madurez.

Pues en efecto, en la medida que el ser humano se desarrolla, a partir de experiencias como la acumulación de recuerdos, la confianza en el ambiente, la comprensión intelectual, entre otros, adquiere recursos propios que lo hacen despojarse del apoyo ambiental (Rodríguez y Oiberman, 2011).

Según Rodríguez y Oiberman, (2016), la importancia de este y otros tipos de instrumentos elaborados con las mismas características, radica en que permiten a los profesionales detectar o prevenir a tiempo síntomas patológicos relacionados con el apego.

\section{Conclusiones}

De acuerdo a la revisión bibliográfica y análisis de los resultados de varias investigación que han marcado precedentes en el estudio del desarrollo intelectual en la primera infancia y otros 
aspectos afines, se ha caracterizado el desarrollo intelectual en la Primera Infancia, puntualizando algunos factores que favorecen el desarrollo cognitivo del niño y otros que lo retrasan.

Se ha señalado que los primeros años de vida resultan fundamentales en el desarrollo del niño, particularmente para nivel cognitivo (Oiberman y Mercado, 2007). Como se ha descrito en los apartados anteriores, el desarrollo cognitivo del niño está fuertemente relacionado o determinado por las experiencias con su medio ambiente a más de la predisposición biológica.

Un aspecto importante del contexto socio cultural es rol de los padres en la adquisición de las habilidades cognitivas de sus hijos. La adecuada estimulación proveniente del medioambiente familiar se asocia a un óptimo desarrollo mental de los niños. Por el contrario si la estimulación que el niño recibe es precaria o inexistente, el desarrollo cognitivo de los infantes será inferior al esperado.

Así mismo, se ha resaltado la importancia y características de instrumentos diseñados para evaluar el desarrollo cognitivo del niño, las estrategias que utiliza en la resolución de algunas situaciones que de acuerdo a la edad y etapa en que se encuentre el niño debe tener. Por ejemplo, en relación con los estudios efectuados por Piaget $(1974,1982)$, los hallazgos realizados por Oiberman y otros $(2002,2005)$, al administrar la EAIS en muestras de niños argentinos, permiten discriminar con mayor especificidad el nivel de las habilidades cognitivas en niños pequeños. Estos resultados permiten, entre otras ventajas, considerar medidas preventivas que disminuyan el impacto de agentes ambientales y sociales en el desarrollo cognitivo óptimo de los niños.

\section{Bibliografia}

Ainsworth, M. D. S., Blehar, M., Waters, E. y Wall, S. (1978). Patterns of attachment: A psychological study of the Strange Situation. New Jersey: Lawrence Erlbaum - an imprint of Taylor \& Francis Group 
Erlbaum. Albornoz, E. y Guzmán, M. (2016). Desarrollo cognitivo mediante estimulación en niños de 3 años: centro desarrollo infantil nuevos horizontes. Quito, ecuador. Revista Universidad y Sociedad, 8(4), 186-192.

Bowlby, J. (1982). El vínculo del niño hacia su madre: la conducta de apego. Madrid. Alianza universal.

Chamorro, L. (2012). El apego. Su importancia para el pediatra. Pediatr, 39(1), 199-206.

Duek, C; (2010). Infancia, desarrollo y conocimiento: los niños y niñas y su socialización. Revista Latinoamericana de Ciencias Sociales, Niñez y Juventud, 8: 799-808.

Evans, G. y English, K. . (2002). The Environment of Poverty: Multiple Stressor Exposure , Psychophysiological Stress, and Socioemotional Adjustment. Child Development, 73(4), 12381248.

Garrido, L. (2006). Apego, emoción y regulación emocional. implicaciones para la salud. Revista Latinoamericana de Psicología, 38(3), 493-507.

Grimalt, L., y Heresi, M. . (2012). Estilos de apego y representaciones maternas durante el embarazo. Chil Pediatr 2, 83(3), 239-246.

Oiberman, A. Laborde, D. ., Fernández, M. ., Macedra, V. ., Zimmermann, E., Barbuto, S. ., y Mujica, C. . (2015). Impacto de los desencuentros tempranos en el desarrollo cognitivo. In UNSL (Ed.), Avaces y Desafíos para la Psicologia (pp. 209-218). San Luis.

Oiberman, A. y Mercado, A. (2007). Nacer, jugar y pensar: guía para acompañar a los bebés. Buenos Aires: Lugar.

Oiberman, A. Orellana, L. y Mansilla, M. . (2006). Evaluación de la inteligencia en bebés argentinos : Escala Argentina de Inteligencia Sensoriomotriz. Arch Argent Pediatr, 104(4), 316324.

Ortiz Soto, P., Duelo Marcos, M., y Escribano Ceruelo, E.. (2013). La entrevista en salud mental infantojuvenil (II): el desarrollo psicoafectivo y cognitivo del niño. Pediatría Atención Primaria, $15(57), 89 \mathrm{e} 41-89 \mathrm{e} 55$

Paolini, C., Santos, M., y Oiberman, A. (2015). Un estudio multicéntrico argentino: Variaciones en el desarrollo cognitivo en bebés nacidos a término. Anuario de Investigaciones, 21, 363-374.

Papalia, D., Wendkos S., y Duskin, R. (2012). Psicologia del Desarrollo. (12ma. ed.). MacGrawHill Interamericana.

Phillips, J. (1977). Los orígenes del intelecto según Jean Piaget. (J. Toro, y R. Berdagué, Trads.) Barcelona, España: Fontanella.

Piaget, J. (1990). La equilibración de las estructuras cognitivas. Madrid: Siglo XXI. 
Piaget, J. y Inhelder, B. ([1969] 2000). Psicología del niño. Madrid, España: Morata.

Rodríguez, G. y Oiberman, A. (2011). Capacidad de estar a solas. In Compendio de investigaciones actuales en psicología y ciencias afines (C.I.I.P.M., pp. 1-12). Buenos Aires.

Rodríguez, G. y Oiberman, A. (2016). Adaptación y Sistematización de una Escala de Apego Para Niños Pequeños. Journal of Latino/Latin-American Studies, 8(2), 59-78.

Schonhaut, L. Maggiolo, M. Herrera, M. Acevedo, K. y García, M. (2008). Lenguaje e inteligencia de preescolares: Análisis de su relación y factores asociados. Rev Chil Pediatr 2008;, 79(6), 600-606.

Stassen, K. (2009). Psicología del desarrollo: Adultez y Vejez. Madrid: Médica Panamericana.

Vargas, J. y Arán, V. (2014). Importancia de la Parentalidad para el Desarrollo Cognitivo Infantil : una Revisión Teórica. Revista Latinoamericana de Ciencias Sociales, Niñez Y Juventud, 1(12), 171-186.

Vargas R. y Arán V. (2014). Importancia de la Parentalidad para el Desarrollo Cognitivo Infantil: una Revisión Teórica. Revista Latinoamericana de Ciencias Sociales, Niñez y Juventud, 12; 171-186. 